\title{
The effect of Garcinia kola extract on lipopolysaccha- ride-induced tissue damage in rats
}

\section{Tebekeme Okoko*, Solomon Ndoni}

Biochemistry Division, Department of Chemical Sciences, Niger Delta University, PMB 71, Wilberforce Island, Bayelsa State, Nigeria.

\begin{abstract}
Purpose: To study the effect of the crude methanol extract of Garcinia kola seed on lipopolysaccharide (LPS)-induced tissue damage in rats

Methods: A total of 16 rats were split equally into four treatment groups: Normal controls, LPS-treated only, LPS-treated $+100 \mathrm{mg} / \mathrm{kg}$ body weight (b.w.) extract, LPS-treated $+250 \mathrm{mg} / \mathrm{kg} \mathrm{b.w}$. extract. LPS was administered via the intraperitoneal route once and the extract orally administered to the rats for 14 days. Indices of liver and kidney function were later assessed.

Results: The results showed that LPS caused marked elevations in the blood levels of alanine aminotransaminase, aspartate aminotransaminase, creatinine, urea, nitric oxide and a significant reduction in the catalase activity in both liver and kidney. The thiobarbituric acid reactive species were also increased in liver. These levels were brought to near control values when the rats were fed with the extract. The changes were more pronounced in the rats given the $250 \mathrm{mg} / \mathrm{kg} \mathrm{b.w.} \mathrm{of} \mathrm{the} \mathrm{extract.}$

Conclusion: The data suggest that Garcinia kola significantly reduced the tissue damage induced by LPS which may be dose-dependent. This is probably due to the interaction of the various phytochemicals with LPS and/or to the fact that the extract possesses significant antioxidant properties.
\end{abstract}

Keywords: Garcinia kola, lipopolysaccharide, creatine, urea, hepatotoxicity, nephrotoxicity.

\footnotetext{
*Corresponding author: E-mail: tebebuddy@yahoo.com
} 


\section{INTRODUCTION}

The use of plants as traditional medicine has been in existence for several years. This practice has been encouraged in recent times due to the less frequent side effects when compared with orthodox medicine. In response to this, the medicinal potentials of a lot of plants have been explored.

Garcinia kola Heckel (Gutifera) is a tropical plant which grows in moist forest. The seeds have a bitter taste hence the plant is commonly called bitter kola as such they are consumed as a stimulant ${ }^{1}$. Extracts of the seeds have been used traditionally for the treatment of cough, and liver diseases ${ }^{2}$. The seeds of the plant have been shown to have various bioactivities in experimental models such as nephroprotective and bronchodilatory effects $^{3,4}$. Various complex compounds have been isolated from Garcinia kola such as kolaviron, xanthones, garcinoic acid, garcinal, and tocotrienol ${ }^{5}$.

Lipopolysaccharide (LPS) is one of the virulence factors of Gram-negative bacteria. Macrophages are among the main targets of LPS and this activity leads to the release of some cytokines and nitric oxide as part of the inflammatory response ${ }^{6}$. Recently, Garcinia kola seed extract was shown to reduce the tendency of lipopolysaccharide to activate macrophages in vitro ${ }^{14}$ however a similar work in an in vivo model has not been reported. This present work looks at the effect of Garcinia kola extract on lipopolysaccharideinduced tissue damage in rats.

\section{MATERIALS AND METHODS}

\section{Chemicals and Reagents}

A vial of lipopolysaccharide (from $E$. coli strain 055:B5) was obtained from Sigma Chemicals (USA). All other chemicals were standard laboratory chemicals and reagents of analytical grade and were used without further purification. Buffers and dilutions were prepared in glass-distilled water unless otherwise stated.

\section{Animals}

Sixteen adult male albino rats (Rattus norvegicus) weighing between 110 - 120g were obtained from the Nigerian Institute of Medical Research, Yaba. They were kept in conventional cages in the animal house owned by the Department of Biological Sciences, Niger Delta University, Wilberforce Island. They were freely given water and standard rat chow ad libitum. Approval for the use of the experimental animals was given by the Ethical Committee for Animal Usage of the Faculty of Science, Niger Delta University.

\section{Preparation of plant extract}

The fruits of Garcinia kola were obtained fresh from the Obunagha forest of Bayelsa State, Nigeria in January 2008. The plant was authenticated by Professor B. L. Nyananyo, a taxonomist in the Department of Plant Science and Biotechnology, University of Port Harcourt, Nigeria. A voucher specimen (P.0126) was deposited in the herbarium unit of the Department of Chemical Sciences, Niger Delta University. The seeds were obtained from the fruits and sun-dried after chopping to smaller bits. They were ground and $500 \mathrm{~g}$ of the resulting powder was soaked in $1 \mathrm{~L}$ of absolute methanol for three days and filtered. The filtrate was later concentrated to dryness using a rotary evaporator set at $40^{\circ} \mathrm{C}$ $\pm 5{ }^{\circ} \mathrm{C}$. The yield was $36.2 \%$. A $0.2 \mathrm{~g} / \mathrm{mL}$ extract was later prepared in distilled water and stored at $4{ }^{\circ} \mathrm{C}$ until required.

\section{Treatment of animals}

The rats were split into four groups $(1,2,3$, and 4). After acclimatizing the rats for six days, rats of groups 2,3 , and 4 were given a single intraperitoneal administration of lipopolysaccharide (1 $\mathrm{mg} / \mathrm{kg}$ body weight) in order to induce tissue damage. Twenty four hours later, rats of groups 3 and 4 were given $100 \mathrm{mg} / \mathrm{kg}$ and $250 \mathrm{mg} / \mathrm{kg}$ body weight daily 
of the extract intragastrically for another 14 days, while group 1 rats were untreated controls.

\section{Collection of tissues}

At the end of the treatment, each rat was given light ether anaesthesia, dissected, the thoracic region opened, blood collected via cardiac puncture and delivered into two tubes - one heparinized and the other containing no anticoagulant. Plasma and serum were collected after allowing the tubes to stand for $10 \mathrm{~min}$, centrifuged and stored at $-4^{0} \mathrm{C}$. The liver and kidney were also excised, washed in ice-cold phosphate buffer $(0.2 \mathrm{M}, \mathrm{pH} 7.5)$, blotted, and $10 \%$ homogenates were prepared using the same buffer. Perinuclear fractions of both liver and kidney were collected by centrifuging homogenates at $5000 \mathrm{rpm}$ for $20 \mathrm{~min}$.

\section{Biochemical analysis}

The levels of alanine aminotransaminase (ALT), and aspartate aminotransaminase (AST) were determined in serum according to the instructions on commercial kits purchased from Randox (UK). Urea and creatinine levels were also analyzed in serum according to the instructions on commercial kits purchased from QCA (Spain). Nitric oxide was determined in plasma according to the method of Hsieh et $a^{\beta}$. Catalase activity was determined by monitoring the consumption of hydrogen peroxide. Briefly, $1 \mathrm{ml}$ of perinuclear fraction, $1.5 \mathrm{ml}$ of distilled water and $1 \mathrm{ml}$ of phosphate buffer $(0.2 \mathrm{M}, \mathrm{pH} 7.5)$ were delivered into a test tube. The reaction was initiated by the addition of $0.5 \mathrm{ml}$ of $0.1 \mathrm{M}$ hydrogen peroxide. The amount of hydrogen peroxide consumed was determined by measuring absorbance at $240 \mathrm{~nm}$ and the catalase activity was expressed as $\mathrm{k} / \mathrm{g}$ tissue. Thiobarbituric acid reactive species (TBARS) in tissues were measured as reported ${ }^{9}$ except that vitamin $\mathrm{C}$ was used as the reducing agent and the reaction took place at room temperature. TBARS was expressed as nanomoles of malondialdehyde (MDA) per gram tissue.

\section{Statistical analysis}

Data are presented as mean \pm S.E.M. Where appropriate, the data were subjected to a twotailed Students' t-test using Minitab statistical software (version 14). A confidence level exhibited at $\mathrm{P}<0.05$ was considered statistically significant.

\section{RESULTS}

The levels of the markers measured in serum and plasma are shown in Table 1. It revealed that the intraperitoneal administration of LPS caused marked elevations in the levels of ALT, AST, urea, creatinine, and nitric oxide. The levels of all these markers were significantly reduced when the Garcinia kola extract was given to the rats. In all cases, the levels of these markers in rats of group 4 are significantly lower than the levels measured in group 3 . The catalase activities and the levels of thiobarbituric acid reactive species (TBARS) in the liver and kidney perinuclear fractions are shown in Table 2. LPS caused significant elevation of TBARS and reduction in the level of catalase in liver. These abnormal levels of the catalase and TBARS were brought close to control values when the extract was administered to the rats. However, the changes were more pronounced in group 4 than group 3. A similar result was also observed for catalase in the kidney but LPS did not cause any change in the level of TBARS in the kidney.

\section{DISCUSSION}

The strong correlation between the elevations in ALT, AST, urea and creatinine indicates that tissue damage which may predispose to lipid peroxidation which is associated with the inflammatory response. It has been demonstrated that LPS significantly increases the production of nitric oxide by the activation of macrophages ${ }^{10}$. Nitric oxide is a signal molecule in the acute inflammatory response 
Table 1: Effect of Garcinia kola extract on enzymatic and non-enzymatic markers in serum and nitric oxide levels in plasma

\begin{tabular}{llllll}
\hline Group & Urea $(\mathbf{m g} / \mathbf{d l})$ & CR $(\mathbf{m g} / \mathbf{d l})$ & ALT $(\mathbf{U} / \mathbf{L})$ & AST $(\mathbf{U} / \mathbf{L})$ & NO $(\mu \mathrm{g} / \mathbf{m l})$ \\
\hline 1 & $15.8 \pm 1.28$ & $1.54 \pm 0.12$ & $13.08 \pm 1.11$ & $26.76 \pm 2.11$ & $1.23 \pm 0.01$ \\
2 & $33.4 \pm 3.21^{*}$ & $4.23 \pm 0.31^{*}$ & $32.12 \pm 0.95^{*}$ & $54.98 \pm 3.12^{*}$ & $23.64 \pm 5.67^{*}$ \\
3 & $21.4 \pm 2.01^{* *}$ & $2.03 \pm 0.45^{* *}$ & $18.44 \pm 1.06^{* *}$ & $36.54 \pm 1.07^{* *}$ & $14.48 \pm 3.76^{* *}$ \\
4 & $17.8 \pm 1.11^{* * \bullet}$ & $1.82 \pm 0.17$ & $15.34 \pm 0.56^{* * \bullet}$ & $31.03 \pm 2.51^{* * \bullet}$ & $5.15 \pm 1.32^{* * \bullet}$
\end{tabular}

Values are presented as means \pm S.E.M $(n=4) .{ }^{*}$ Significantly different from group $1 ;{ }^{*}$ significantly different from group 2, and significantly different from group $3(\mathrm{P}<0.05)$. CR, ALT, AST, NO (Creatinine, Alanine aminotransaminase, aspartate aminotransaminase, nitric oxide respectively)

Table 2: Effect of Garcinia kola extract on the levels of catalase and TBARS in liver and kidney

\begin{tabular}{|c|c|c|c|c|}
\hline \multirow[t]{2}{*}{ Group } & \multicolumn{2}{|c|}{ Liver } & \multicolumn{2}{|c|}{ Kidney } \\
\hline & Cat $(\mathbf{k} / \mathbf{g})$ & TBARS & Cat $(\mathbf{k} / \mathbf{g})$ & TBARS \\
\hline 1 & $42.12 \pm 2.61$ & $24.36 \pm 1.87$ & $52.12 \pm 5.76$ & $19.62 \pm 0.43$ \\
\hline 2 & $23.86 \pm 3.54^{*}$ & $42.55 \pm 2.08^{*}$ & $21.54 \pm 2.96^{*}$ & $21.25 \pm 0.67$ \\
\hline 3 & $36.23 \pm 3.08^{* \#}$ & $37.21 \pm 2.67^{* \#}$ & $37.28 \pm 2.31^{* \#}$ & $20.02 \pm 1.01$ \\
\hline 4 & $39.65 \pm 2.42^{* \# \bullet}$ & $30.48 \pm 2.21^{* \# \bullet}$ & $42.58 \pm 3.06^{* \# \bullet}$ & $20.11 \pm 1.09$ \\
\hline
\end{tabular}

Values are presented as means \pm S.E.M $(n=4)$. * Significantly different from group 1 ; " significantly different from group 2, and "significantly different from group $3(P<0.05)$.

Cat, TBARS (catalase, thiobarbituric acid reactive species respectively). TBARS was expressed as nmol $M D A / g$ tissue.

which is derived from the oxidation of $L$ arginine catalyzed by nitric oxide synthase ${ }^{11}$. The results reveal that LPS is both hepatotoxic and nephrotoxic. This may give insight into the pathogenesis of some infectious diseases that are local to the liver and kidney as LPS is a virulence factor of Gram-negative bacteria. The increase in the level of TBARS in the liver following the administration of LPS indicates the activation of the lipid peroxidation system which is often initiated by the abstraction of hydrogen atoms from membrane lipids. Now, it is known that the measurement of TBARS in tissues is a method of choice for monitoring lipid peroxidation which is a major indicator of oxidative stress ${ }^{12}$. Although, high levels of TBARS were not observed in kidney when the rats were treated with LPS, the elevations in urea and creatinine confirm the onset of kidney damage.

The fact that the methanolic extract of Garcinia kola reduced the damage caused by LPS buttresses the protective role of Garcinia kola in tissues against free radical damage whether from a chemical or microbial source. This work also elucidates some manifestations of the damaging effects of LPS.

The protective virtues of Garcinia kola have been ascribed to the presence of biflavonoid compounds ${ }^{5}$. Much interest has focused on flavonoids - a group of polyphenols. Flavonoids such as kolaviron have the 
propensity of improving endothelial function by lowering oxidative stress which predisposes to improvement of coronary circulation ${ }^{13}$.

Gram-negative bacteria mostly contain (> $90 \%$ ) of lipopolysaccharide on the surface of the outer membrane and is thought to be the protective wall rendering Gram-negative bacteria resistant to a variety of intrinsic host defence molecules ${ }^{14}$. Thus this implies that the reduction in the tissue damage caused by LPS may be attributed to direct interaction with the phytochemicals.

\section{CONCLUSION}

This work further illustrates one of the ways by which gram-negative bacteria can cause damage to tissues and the probable mechanism of the reported antimicrobial properties of Garcinia kola extract. Based on these findings, the controlled consumption of Garcinia kola is recommended. The presence of Garcinia kola in the biosphere is nature's gift to mankind as a source of abundant nutrients. Progress in this area will be of immense pharmaceutical interest.

\section{REFERENCES}

1. Atawodi S, Mende P, Pfundstein B, Preussmann R, Spiegelhalder $B$. Nitrostable amines and nitrosamide formation in natural stimulants, Cola acuminate, Cola nitida, and Garcinia kola. Food Chem Toxicol, 1995; 33: 625-630.

2. Braide VD. Pharmacology effects of chronic injection of Garcinia kola seeds in the rat. Phytother Res, 1991; 4: 39-41.

3. Okoko T, Awhin EP. Garcinia kola extract reduced cisplatin-induced kidney dysfunction in rats. Afr $J$ Biochem Res, 2007; 1: 124-126.
4. Orie NN, Ekon EU. The bronchodilatory effects of Garcinia kola. Afr J Pharm Pharm Res, 1993; 22: 172-201.

5. Terashima $K$, Takawa $Y$, Niwa $M$. Powerful antioxidant agents based on garcinoic acid from Garcinia kola. Bioorg Med Chem, 2002; 10: 16191625.

6. Meng F, Lowell CA. Lipopolysaccharide-induced macrophage activation and signal transduction in the absence of scr family kinases Hck, Fgr, and Lyn. J Exp Med, 1997; 189: 1661-1670.

7. Okoko T, Oruambo IF. Garcinia kola extract reduced lipopolysaccharide activation of macrophages using U937 cells as a model. Afr J Biotech, 2008, 7: 792-795.

8. Hsieh YH, Kuo PM, Chien SC, Shyur LF, Wang SY. Effects of Chamaecyparis formosensis Matasumura extractivities on lipopolysaccharideinduced release of nitric oxide. Phytomed, 2007; 14: $675-680$

9. McDonald RE, Kelleher SD, Hultin HO. Membrane lipid peroxidation in a microsomal fraction of red hake muscle. J Biochem, 1979; 3: 125-134.

10. Park YC, Kimback G, Salion C, Valacchi G, Parcker L. Activity of monomeric, dimeric, and trimeric flavonoids on NO production, TNF- $\alpha$ secretion, and NF-kB dependent gene expression in RAW264.7 macrophages. FEBS Lett, 2000; 465: 93-97.

11. Surh YJ, Chun KS, Cha HH, Han SS, Keun YS, Park $K K$, Lee SS. Molecular mechanisms underlying chemoprotective activities of anti-inflammatory phytochemicals; down regulation of cox-2, and iNOS through suppression of NF-kB activation. Mutat Res, 2001; 480: 243-268.

12. Yagi K. Simple procedure for specific assay of lipid hydroperoxides in serum and plasma. Methods Mol Biol, 1998; 108: 107-110.

13. Hirata K, Shimada K, Watanabe H, Otsuka R, Tokai $K$, Yoshiyama $M$ et al. Black tea increases coronary flow rate velocity reserve in healthy male subjects. Am J Cadiol, 2004; 93: 1384-1388.

14. Nikaido $H$. Prevention of drug access to bacterial targets: permeability barriers and active efflux. Science, 1994; 264: 382-388. 
Okoko \& Ndoni

Trop J Pharm Res February 2009; 8 (1): 32 